Hill, R.W., Huelsman, T. J., \& Araujo, G. (2010). Perfectionistic concerns suppress associations between perfectionistic strivings and positive life outcomes. Personality and Individual Differences. 48(5): $584-589$ (April 2010). Published by Elsevier (ISSN: 0191-8869).

http://0-dx.doi.org.wncln.wncln.org/10.1016/j.paid.2009.12.011

\title{
Perfectionistic concerns suppress associations between perfectionistic strivings and positive life outcomes
}

Robert W. Hill, Timothy J. Huelsman, Gustavo Araujo

\begin{abstract}
Perfectionism has been frequently associated with diverse negative psychological outcomes, but less frequently with positive psychological outcomes. This investigation reports multiple regression analyses based on survey data from 216 psychology students. These analyses indicate that adaptive perfectionistic strivings and maladaptive perfectionistic concerns predict desirable outcomes including psychological well-being, life satisfaction, and affect. That perfectionistic strivings predicted these outcomes is inconsistent with the small zero-order correlations between perfectionistic strivings and the positive outcomes, indicating that maladaptive perfectionistic concerns act as a suppressor variable in this relationship. These findings illustrate the importance of conceptualizing perfectionism as having both adaptive and maladaptive dimensions, and of assessing both simultaneously when examining relationships between perfectionism and other substantive variables.
\end{abstract}




\section{INTRODUCTION}

While perfectionism was initially proposed as a unidimensional construct (Burns, 1980), more recent investigation has described it as a multidimensional construct that includes having high personal standards or striving for excellence, having concerns about making mistakes, having high standards for others, having concern for the approval of others for one's performance, being neat, and having a tendency toward obsessive rumination (Hill et al., 2004). Perfectionism has been assessed using several multidimensional instruments available in the research literature (Frost et al., 1990, Hewitt and Flett, 1991 and Hill et al., 2004), but factor analyses reveal that virtually any measure of perfectionism reflects two basic forms of perfectionism (see Stoeber \& Otto, 2006, for a comprehensive review). These two forms are called perfectionistic strivings and perfectionistic concerns, respectively, by Stoeber and Otto (2006), but they are variously referred to as adaptive and maladaptive perfectionism (Rice, Ashby, \& Slaney, 1998), positive striving and maladaptive evaluative concerns (Frost, Heimberg, Holt, Mattia, \& Neubauer, 1993), healthy and unhealthy perfectionism (Stumpf \& Parker, 2000), conscientious and self-evaluative perfectionism (Hill et al., 2004), and positive and negative perfectionism (Terry-Short, Owens, Slade, \& Dewy, 1995). This lack of consistency in terms is undesirable; thus, following Stoeber and Otto's advice, we will use the terms perfectionistic strivings and perfectionistic concerns throughout this manuscript.

Perfectionistic characteristics have been related to a broad variety of negative outcomes including depression, social anxiety and social phobias, obsessive-compulsive disorder, eating disorders, somatic complaints, and maladaptive personality characteristics (for detailed reviews see Flett and Hewitt, 2002, Shafran and Mansell, 2001 and Stoeber and Otto, 2006). Though perfectionism has been frequently studied in association with undesirable outcomes, it has been linked to desirable outcomes less often. Nonetheless, a growing literature has related perfectionism to more positive psychological variables, recently summarized by Stoeber and Otto. Despite these reports, some investigators have expressed doubts that perfectionism can be positive, adaptive, or healthy (Flett and Hewitt, 2002, Flett and Hewitt, 2005 and Greenspon, 2000).

In their review of the positive aspects of perfectionism, Stoeber and Otto (2006) summarized a variety of positive psychological outcomes associated primarily with various representations of perfectionistic strivings. They presented diverse findings and suggested that more weight should be given to the investigation of positive outcomes, especially those that do not overlap with the scales used to measure perfectionistic strivings. The literature on positive versus negative characteristics associated with perfectionism has been complicated by the use of differing conceptualizations of perfectionism, as well as some mixed results associating both positive and negative characteristics with positive striving perfectionism (Bieling et al., 2004 and Bieling et al., 2003). Additionally some investigators have utilized a dimensional approach to assessing associations with perfectionism, as in the current investigation of perfectionism factors related to positive life outcomes, while others have identified groups of perfectionists, often described as healthy or unhealthy, and compared them on various outcomes (see Stoeber \& Otto, 2006). Following the Stoeber and Otto review, the current investigation had the goal of examining the relationship of both perfectionistic strivings and perfectionistic concerns dimensions with indices 
of positive psychological functioning. In particular, this investigation used the Perfectionism Inventory (PI; Hill et al., 2004), a multidimensional measure of perfectionism, with perfectionistic strivings and perfectionistic concerns factors (called conscientious perfectionism and selfevaluative perfectionism, respectively), to assess associations with measures of psychological well-being, satisfaction with life, and mood. This particular measure was chosen because of its two-dimensional structure and because it incorporates the strengths of other measures of perfectionism while avoiding some of their weaknesses (see Hill et al. for a thorough review of these issues). Thus, this study was designed to advance the literature associating perfectionism with positive outcomes.

\subsection{Psychological well-being}

According to Ryff (1989), psychological well-being represents more than mere happiness. Rather, constructs such as sense of purpose and direction, self-realization, and achievement of satisfying relationships are all parts of psychological well-being. Thus, she advocated for a model of psychological well-being comprising six dimensions of positive psychological functioning: autonomy, environmental mastery, personal growth, positive relations with others, purpose in life, and self-acceptance. In an investigation of the relationship between perfectionism and this conceptualization of psychological well-being, Chang (2006a) found strong negative zero-order associations between all six psychological well-being scales and perfectionistic concerns, with self-appraised stress mediating the inverse correlation. However, perfectionistic strivings was positively correlated with only purpose in life and personal growth. In another investigation associating positive outcomes with perfectionism, Chang (2006b) utilized a more complex conceptual conceptualization of perfectionism that included outcome cognition valence. While Chang, 2006a and Chang, 2006b has described some intriguing (though mixed) relationships between perfectionism factors and psychological well-being, the current study is designed to clarify and substantiate the relationship between perfectionism and psychological well-being by using the dimensions of perfectionistic strivings and perfectionistic concerns. We hypothesized that perfectionistic strivings would be associated positively and perfectionistic concerns would be associated negatively with each of Ryff (1989) indices of psychological well-being.

\subsection{Life satisfaction}

Another positive psychological outcome with which perfectionism may be related is life satisfaction. One influential perspective of life satisfaction defined it as "a judgmental process in which individuals assess the quality of their lives on the basis of their own unique set of criteria" (Pavot \& Diener, 1993, p. 164). In other words, people compare their life circumstances with their self-derived standards and report life satisfaction with respect to the degree that these perceived life conditions match these self-standards. Chang (2000) found an inverse relationship between a composite measure of general perfectionism and satisfaction with life in both young and middle aged adults. Chang (2006b) later reported mixed results using more complex perfectionism scales, although his constructs only partly overlap with the more common perfectionistic strivings and perfectionistic concerns used in this investigation. In another investigation of perfectionism in adolescents, Gilman and colleagues reported positive 
associations between perfectionistic striving and life satisfaction and negative associations between perfectionistic concerns and life satisfaction (Gilman and Ashby, 2003 and Gilman et al., 2005). For the current investigation, we sought to clarify further the relationship between life satisfaction and perfectionism, hypothesizing that perfectionistic striving would be associated positively with life satisfaction and perfectionistic concerns would be associated negatively with life satisfaction.

\subsection{Affect}

Another measure of positive psychological functioning is affect. The experience of positive versus negative affect can serve as a positive psychological outcome indicator, or an index of happiness (Ryff, 1989). When assessing the association between composite perfectionism and affect, Chang (2000) found that composite perfectionism was related to negative affect (but not positive affect), but the relationship was mediated by stress (rather than perfectionism leading directly to negative affect). In a later investigation, Chang (2006b) reported mixed results using more complex constructs than the current conceptualization of perfectionistic strivings and concerns. Further, he did not report associations with negative affect. In an investigation of perfectionism and health, Molnar, Reker, Culp, Sadava, and DeCourville (2006) found a significant positive association between perfectionistic striving and positive affect. Molnar and colleagues also reported that perfectionistic concerns were inversely related to positive affect and positively related to negative affect. Further, their results suggested that affect played a mediating role in the relationship between perfectionism and self reported physical health (rather than perfectionism directly leading to health outcomes). Dunkley, Zuroff, and Blankstein (2003) found that negative affect (recorded on a daily basis for a week) was negatively related to perfectionistic striving and positively related to perfectionistic concern, and that positive affect was negatively related to perfectionistic concern. For the current investigation, we hypothesized that perfectionistic striving would be positively associated with positive affect and negatively associated with negative affect. Similarly, we hypothesized that perfectionistic concern would be negatively associated with positive affect but positively associated with negative affect.

\section{METHOD}

\subsection{Participants}

Participants were recruited from Psychology classes and earned course credit for their participation. After granting informed consent, participants anonymously completed each measure in counterbalanced order in 1-h group sessions. The study received Institutional Review Board approval and was conducted in accordance with ethical standards of research with human participants. Two hundred and sixteen participants, 116 females and 83 males (17 respondents did not report) participated in the study. The participants ranged in age from 18 to $25(M=19.87, S D=1.41)$. Ethnicity was not recorded in the current study, but the university from which participants were obtained has a student body that is $92 \%$ Caucasian and $3.2 \%$ African-American (4.8\% of students report other ethnicities). 


\subsection{Measures}

\subsubsection{Perfectionism}

The Perfectionism Inventory (PI; Hill et al., 2004) is an empirically derived self-report measure of perfectionism. The PI comprises 59 items distributed on eight scales with coefficient alphas ranging from .83 to .91. Coefficient alphas for the current data are reported in Table 1 . The eight scales of the Perfectionism Inventory are concern over mistakes, high standards for others, need for approval, organization, perceived parental pressure, planfulness, rumination, and striving for excellence. The PI yields three composite scores. The PI Composite includes a sum of all PI scales and represents a general perfectionism score. The Conscientious Perfectionism composite score is derived from the sum of the following scales: organization, striving for excellence, planfulness, and high standards for others. The self-evaluative perfectionism composite score is derived from the sum of the following scales: concern over mistakes, need for approval, rumination, and perceived parental pressure. In this investigation the Conscientious Perfectionism composite was used to assess perfectionistic strivings, and the self-evaluative perfectionism composite was used to assess perfectionistic concerns.

\begin{tabular}{|c|c|c|c|c|c|c|c|c|c|c|c|}
\hline & 1 & 2 & 3 & 4 & 5 & 6 & 7 & 8 & 9 & 10 & 11 \\
\hline $\begin{array}{l}\text { 1. Perfectionistic strivings } \\
\text { 2. Perfectionistic concerns }\end{array}$ & $\begin{array}{l}(.92) \\
.55\end{array}$ & $(.94)$ & & & & & & & & & \\
\hline $\begin{array}{l}\text { 3. Autonomy } \\
\text { 4. Environmental mastery }\end{array}$ & $\begin{array}{r}-.01^{\mathrm{b}} \\
.16^{2}\end{array}$ & $\begin{array}{l}-.37 \\
-.39\end{array}$ & $\begin{array}{l}(.82) \\
.44\end{array}$ & $(.83)$ & & & & & & & \\
\hline 5. Personal growth & $.06^{\mathrm{b}}$ & -.27 & .52 & .52 & $(.84)$ & & & & & & \\
\hline 6. Purpose in Life & .30 & $-.16^{2}$ & .42 & .65 & .60 & $(.79)$ & & & & & \\
\hline 7. Self-acceptance & $.06^{\mathrm{b}}$ & -.45 & .52 & .78 & .60 & .69 & $(.89)$ & & & & \\
\hline 8. Positive relations & $-.07^{\mathrm{b}}$ & -.44 & .36 & .62 & .57 & .55 & .68 & $(.87)$ & & & \\
\hline 9. Satisfaction with life & $.15^{2}$ & -.25 & .25 & .63 & .46 & .59 & .71 & .56 & $(.89)$ & & \\
\hline 10. PAUD & $.11^{\mathrm{b}}$ & -.16 & .38 & .51 & 33 & .37 & .47 & .38 & .41 & $(.88)$ & \\
\hline 11. UAPD & $.02^{\mathrm{b}}$ & .35 & -.23 & -.53 & -.33 & -31 & -.55 & -.46 & -.46 & -.43 & $(.89)$ \\
\hline$M$ & 12.32 & 10.82 & 60.72 & 59.80 & 68.34 & 61.89 & 63.31 & 64.92 & 23.81 & 31.44 & 26.45 \\
\hline$S D$ & 2.71 & 2.98 & 10.14 & 9.63 & 9.30 & 8.94 & 11.49 & 11.62 & 6.18 & 5.23 & 7.03 \\
\hline
\end{tabular}

Hill et al. (2004) report that the PI has good convergent validity with other measures of perfectionism such as the Multidimensional Perfectionism Scales by Hewitt and Flett, 1991 and Frost et al., 1990. All PI scales have good variability and clear unidimensional structures, as reflected in exploratory principal components analyses, confirmatory factor analysis, and internal consistency. Test-retest correlations for the eight PI scales ranged from .71 to .91 over four to five weeks (Hill et al., 2004).

\subsubsection{Psychological well-being}

The Scales of Psychological Well-Being (SPWB; Ryff, 1989) consists of six 14-item scales constructed to assess the dimensions of autonomy, environmental mastery, personal growth, 
positive relations with others, purpose in life, and self-acceptance. Ryff (1989) reported alpha coefficients for the scales ranging from 0.86 to 0.93 , and test-retest reliability over a 6 -week period ranging from 0.81 to 0.88 . Coefficient alphas for the current data are reported in Table 1. The scales correlated positively with established measures of positive functioning such as life satisfaction, affect balance, and self-esteem (Ryff, 1989).

\subsubsection{Life satisfaction}

The Satisfaction with Life Scale (SWLS; Diener, Emmons, Larsen, \& Griffin, 1985) is a five-item measure that assesses a person's global judgment of life satisfaction. The SWLS has shown strong inter-item reliability (coefficient alpha of .87), a 2-month test-retest stability coefficient of .82 and a four-year test-retest stability rate of .54 as reported in a review of the scales conducted by Pavot and Diener (1993). Coefficient alphas for the current data are reported in Table 1. This measure has also demonstrated discriminant validity with other scales of emotional well-being (Pavot \& Diener, 1993).

\subsubsection{Affect}

The Four Dimensional Mood Scale (4DMS; Huelsman, Nemanick, \& Munz, 1998) consists of 20 adjectives and is designed to measure pleasant activation, unpleasant deactivation, unpleasant activation, and pleasant deactivation. Participants rated each adjective on the extent to which it generally described their mood using a 5-point rating scale. Data supporting the reliability and validity of the 4DMS may be found in Huelsman et al. (1998) and in Huelsman, Furr, and Nemanick (2003). Coefficient alphas for the current data are reported in Table 1.

\section{RESULTS}

Descriptive statistics for all predictors (perfectionistic strivings and perfectionistic concerns) and criteria (psychological well-being: autonomy, environmental mastery, personal growth, purpose in life, self-acceptance; life satisfaction; affect: pleasant activation-unpleasant deactivation, unpleasant activation-pleasant deactivation) appear in Table 1. Skew and kurtosis are not reported, but there were no notable deviations from normality for any of the study variables. Internal consistency reliability coefficients were generally high ( $\alpha$ ranging from .79 to .94). The correlation between the two predictor variables was rather strong $(r=.55)$, but perfectionistic concerns had stronger zero-order relationships with the criterion variables (mean $|r|=.32$; all $r s$ were statistically significant) than did perfectionistic striving (mean $|r|=.10$; only 4 of 9 rs were statistically significant). The correlations among the PWBS scales were quite strong (mean $r=.57$ ). Similarly, the life satisfaction and affect scales (pleasant activation-unpleasant deactivation, unpleasant activation-pleasant deactivation) also were strongly related to the other criterion variables (mean $|r|=.38$ ).

\subsection{Tests of the hypotheses}

Separate standard multiple regression analyses (see Table 2) were performed for each of the nine criterion variables, regressing the positive outcome on perfectionistic striving and 
perfectionistic concerns (predictors were entered simultaneously). Evaluation of assumptions did not require any transformation of the data, or the deletion of any outliers.

\begin{tabular}{|c|c|c|c|c|c|c|c|c|c|}
\hline \multirow[t]{2}{*}{ Criterion } & \multicolumn{3}{|c|}{ Model } & \multicolumn{3}{|c|}{ Perfectionistic strivings } & \multicolumn{3}{|c|}{ Perfectionistic concerns } \\
\hline & $\overline{R^{2}}$ & $F$ & Constant & B & $r$ & $s r^{2}$ & B & $r$ & $s r^{b}$ \\
\hline Autonomy & .191 & 25.10 & 67.20 & 1.03 & -.01 & .23 & -1.77 & -.37 & -.44 \\
\hline Environmental mastery & 345 & 55.90 & 60.19 & 1.88 & .16 & .44 & -2.18 & -.39 & -.57 \\
\hline Personal growth & .133 & 16.25 & 70.09 & 1.03 & .06 & .25 & -1.34 & -.27 & -.36 \\
\hline Purpose in life & .233 & 32.15 & 20.61 & 1.81 & .30 & .46 & -1.36 & -.16 & -.38 \\
\hline Self-acceptance & 341 & 54.87 & 68.98 & 1.89 & .06 & 37 & -2.67 & -.45 & -.58 \\
\hline Positive relations & .233 & 32.23 & 75.88 & $1.07^{\circ}$ & -.07 & .21 & -2.23 & -.44 & -.48 \\
\hline Satisfaction with life & .174 & 22.27 & 22.84 & 0.92 & .15 & 34 & -0.97 & -.25 & -.39 \\
\hline PAUD & .079 & 9.09 & 30.69 & $0.54^{\circ}$ & .11 & .23 & -0.54 & -.16 & -.26 \\
\hline UAPD & .164 & 20.93 & 21.93 & $-0.64^{\circ}$ & .02 & -.21 & 1.14 & 35 & .41 \\
\hline
\end{tabular}

For all multiple regressions, the multiple $R^{2}$ values were statistically significant, ranging from .08 for pleasant activation-unpleasant deactivation to .35 for environmental mastery. Further, the regression weights for both perfectionistic striving and perfectionistic concerns were statistically significant and in the predicted directions for each of the positive outcomes. Specifically, we found positive relationships between perfectionistic strivings and the criteria, and negative relationships between perfectionistic concerns and the criteria (except unpleasant activationpleasant deactivation which was in the opposite direction, as predicted, for each regression).

That the regression weights were statistically significant for both perfectionistic striving and perfectionistic concerns is remarkable considering that the zero-order correlations between perfectionistic striving and the positive outcomes were generally quite small; 6 of 9 correlations were not statistically significant and only the correlation with purpose in life $(r=.30)$ was greater than .16. This represents a situation of classical suppression (Tabachnick \& Fidell, 2007) in which perfectionistic concern served as a suppressor since by removing variance due to perfectionistic concern, prediction of the positive outcome by perfectionistic striving was enhanced. This is further demonstrated by comparing the zero-order correlations $(r)$ to the semipartial correlations ( $s r$ ) in Table 2. The value of $s r$ for perfectionistic striving was dramatically improved relative to $r$, whereas these two values were more similar for perfectionistic concern.

\section{DISCUSSION}

These results reflect significant associations between the perfectionism dimensions of perfectionistic striving and perfectionistic concern and the outcomes of psychological well-being, life satisfaction, and mood. As suggested by Stoeber and Otto (2006) in their review, the current analyses separated the variance assessed by these two correlated perfectionism dimensions 
and revealed a strong pattern with perfectionistic concern serving as a suppressor variable that reduced the strength of associations between perfectionistic striving and positive psychological outcome variables.

\subsection{Perfectionistic striving and positive outcomes}

The zero-order correlations between perfectionistic striving and psychological well-being scales reflected negligible associations, with the exception of a strong correlation with purpose in life. When variance due to perfectionistic concern was partialled out of the correlations between perfectionistic striving and psychological well-being scales, strong positive relationships were revealed, indicating a suppressor role for perfectionistic concerns.

Perfectionistic striving is a composite of four separate PI scales including striving for excellence, organization, planfulness, and having high standards for others. These dimensions are arguably adaptive and have good face validity in educational, work, and domestic milieus. The strong associations with the psychological well-being scales suggest a relationship between perfectionistic striving and these diverse indicators of well-being. Ryff (1989) argued that these dimensions of well-being, particularly self-acceptance and environmental mastery, were strongly associated with measures of life satisfaction, affect balance, self-esteem, and morale. Ryff suggested that an individual's sense of purpose and direction, self-realization, and achievement of satisfying relationships, as measured by psychological well-being scales, are better predictors of a comprehensive view of psychological well-being as opposed to simple happiness. The results of this investigation support the conception of perfectionistic striving as adaptive, via the strong association with a multidimensional assessment of psychological well-being.

Perfectionistic striving was also positively associated with satisfaction with life, which involves the assessment of one's life circumstances with respect to a match with self-imposed standards, e.g., "My life is close to my ideal; I have gotten the important things I want in life." Both perfectionistic striving and satisfaction with life involve a reflection of internal standards and cognitive judgments that appear adaptive in nature. The more a person endorses having high internal standards for performance for themselves and others, and values being neat and organized, the more likely they are to be satisfied with life.

Similarly, and as predicted, perfectionistic striving was also strongly associated with positive mood, and inversely associated with negative mood. The mood scales measured both the affect dimension of valence (pleasant vs. unpleasant mood) and the affect dimension of arousal (activation vs. deactivation). As levels of perfectionistic striving rise an individual is more likely to be pleasantly activated (e.g., excited) and less likely to be unpleasantly deactivated (e.g., lethargic); the inverse association applies for perfectionistic concerns. As levels of perfectionistic concern rise an individual is more likely to be unpleasantly activated (e.g., irritated) and less likely to be pleasantly deactivated (e.g., serene); again, the inverse association applies for perfectionistic striving. Thus, the current data demonstrate that perfectionism has strong association with positive psychological outcomes that involve affect. In summary, the more an individual endorses having high standards, being organized and planful, and having high standards for others, the more they endorse having high psychological well-being, positive mood, and satisfaction with life. 


\subsection{Perfectionistic concerns and positive outcomes}

The results of this investigation also revealed strong negative zero-order associations between perfectionistic concerns and indicators of psychological well-being, life satisfaction, and positive mood. When the variance due to perfectionistic striving was partialled out, the negative associations grew stronger, and the regression results reflect significant negative associations with all psychological well-being scales, satisfaction with life, and positive affect. Perfectionistic concern is a composite of concern over mistakes, need for approval, rumination, and perceived parental pressure. These perfectionism dimensions involve experiencing anxiety about doing things incorrectly, failing to meet standards, being judged by others, and worrying about performance. The results provide evidence that these perfectionism behaviors can be maladaptive, as reflected in the strong negative associations with all psychological well-being scales and satisfaction with life. Additionally, perfectionistic concern was inversely associated with positive affect, and positively associated with negative affect. Perfectionistic concern was associated with diverse negative outcomes in this investigation suggesting this perfectionism dimension is maladaptive. Additionally, the current analyses revealed that perfectionistic concern suppresses the associations between perfectionistic striving and positive outcomes. Partial correlations and regression analyses were useful in detecting the strength of actual associations between perfectionistic striving and perfectionistic concern dimensions and psychological outcomes.

\subsection{Accounting for the suppressor relationship}

The current investigation thus supports and extends previous findings describing the multidimensional nature of perfectionism, specifically linking perfectionistic striving dimensions with positive life outcomes, and perfectionistic concern dimensions with inverse associations (Chang, 2000, Chang, 2006a, Chang, 2006b, Dunkley et al., 2003, Gilman and Ashby, 2003 and Molnar et al., 2006). This investigation highlights the importance of partialling out variance to adjust for the correlation between the perfectionistic striving and perfectionistic concern factors. The results also demonstrate the need for quantitative strategies beyond zeroorder correlations and simple regression analyses when assessing the association between perfectionism factors and suspected outcomes. Even though perfectionistic striving and perfectionistic concern are positively correlated, as are other indices of adaptive versus maladaptive perfectionism, variance needs to be separately assessed for these perfectionism factors to accurately portray relationships with other psychological outcomes.

In order to assess for a possible interaction effect between perfectionistic striving and perfectionistic concern moderated multiple regression analyses were performed, but when the interaction was added in the second step of the regression analyses no statistically significant additional variance to $R^{2}$ was added for any of the positive outcome criterion variables. Perfectionism investigators are advised to assess for a suppressor relationship, particularly perfectionistic concerns suppressing associations with perfectionistic striving, when assessing the relative associations with positive and negative perfectionism dimensions, and to avoid using a composite measure of general perfectionism. A lack of significant zero-order correlation 
between perfectionistic striving and criterion variables may be the consequence of a suppressor relationship caused by perfectionistic concerns.

\subsection{Implications and summary}

The suppressor effect found here has implications for researchers, but it also has implications for practitioners in clinical, school, and work settings. Even though previous research may not reveal them, perfectionistic strivings and perfectionistic concerns each may have relationships with important outcomes, if one assesses with appropriate analyses. Thus, we reiterate the advice to examine perfectionistic strivings and perfectionistic concerns for suppressor effects. The practical implications of the associations between perfectionistic striving and positive psychological outcomes support the development of interventions to assist individuals who report lower levels of psychological well-being, life satisfaction, and positive affect, and higher levels of negative affect. Based on the results of this investigation, interventions that promote perfectionistic striving, including cognitive processes that involve developing increased organization and planning while encouraging the development of internalized standards for achievement, could be associated with improved positive psychological outcomes. Additionally, individuals who experience high levels of perfectionistic concerns may benefit from interventions designed to reduce their tendencies to worry about mistakes and expect negative appraisals of performance from others, since high levels of perfectionistic concerns appear to handicap an individual in experiencing psychological well-being, satisfaction, and happiness.

The findings of this investigation may be limited in generalizability due to the lack of representativeness of the student sample. Future research should seek to further substantiate the associations between perfectionism and positive psychological outcomes with older, more demographically diverse samples. It may also be fruitful to examine more specific dimensions of both perfectionistic strivings and perfectionistic concerns in future research. In addition, given the reduced levels of positive psychological outcomes associated with perfectionistic concerns, as well as the numerous symptoms of psychopathology previously mentioned, a pre-post investigation of the effects of an intervention to reduce levels of perfectionistic concerns, and associated change in positive psychological outcomes may be of interest. Similarly, interventions designed to increase levels of perfectionistic striving might be assessed for their impact on positive psychological outcomes, as well as other performance outcomes in school and work settings. Evidence that dimensions of perfectionism are adaptive and desirable is accumulating, and investigators need to employ analyses to account for the suppression of perfectionistic striving by perfectionistic concerns.

\section{REFERENCES}

Bieling, P. J., Israeli, A., \& Antony, M. M. (2004). Is perfectionism good bad or both? Examining models of the perfectionism construct. Personality and Individual Differences, 36, 1373-1385. 
Bieling, P. J., Israeli, A., Smith, J., \& Antony, M. M. (2003). Making the grade: The behavioral consequences of perfectionism in the classroom. Personality and Individual Differences, 35, 163-178.

Burns, D. D. (1980). The Perfectionist's script for self-defeat. Psychology Today(November), 70-76.

Chang, E. C. (2000). Perfectionism as a predictor of positive and negative psychological outcomes: Examining a mediation model in younger and older adults. Journal of Counseling Psychology, 47, 18-26.

Chang, E. C. (2006a). Perfectionism and dimensions of psychological well-being in a college student sample: A test of a stress mediation model. Journal of Social and Clinical Psychology, 25, 1001-1022.

Chang, E. C. (2006b). Conceptualization and measurement of adaptive and maladaptive aspects of performance perfectionism: Relations to personality, psychological functioning, and academic achievement. Cognitive Therapy Research, 30, 677-697.

Diener, E., Emmons, R. A., Larsen, R. J., \& Griffin, S. (1985). The satisfaction with life scale. Journal of Personality Assessment, 49, 71-75.

Dunkley, D. M., Zuroff, D. C., \& Blankstein, K. R. (2003). Self-critical perfectionism and daily affect: Dispositional and situational influences on stress and coping. Journal of Personality and Social Psychology, 84, 234-252.

Flett, G. L., \& Hewitt, P. L. (2002). Perfectionism and maladjustment: An overview of theoretical, definitional, and treatment issues. In G. L. Flett \& P. L. Hewitt (Eds.), Perfectionism: Theory, research, and treatment (pp. 5-13). Washington, DC: American Psychological Association.

Flett, G. L., \& Hewitt, P. L. (2005). The perils of perfectionism in sports and exercise. Current Directions in Psychological Science, 14, 14-18.

Frost, R. O., Heimberg, R. G., Holt, C. S., Mattia, J. I., \& Neubauer, A. L. (1993). A comparison of two measures of perfectionism. Personality and Individual Differences, 14, 119-126.

Frost, R. O., Marten, P., Lahart, C., \& Rosenblate, R. (1990). The dimensions of perfectionism. Cognitive Therapy and Research, 14, 449-468.

Gilman, R., \& Ashby, J. S. (2003). A first study of perfectionism and multidimensional life satisfaction among adolescents. Journal of Early Adolescence, 23, 218-235.

Gilman, R., Ashby, J. S., Sverko, D., Florell, D., \& Varjas, K. (2005). The relationship between perfectionism and multidimensional life satisfaction among Croatian and American youth. Personality and Individual Differences, 39, 155-166. 
Greenspon, T. S. (2000). "Healthy perfectionism" is an oxymoron! Reflections on the psychology of perfectionism and the sociology of science. Journal of Secondary Gifted Education, 11, 197-208.

Hewitt, P. L., \& Flett, G. L. (1991). Perfectionism in the self and social contexts: Conceptualization, assessment, and association with psychopathology. Journal of Personality and Social Psychology, 60, 456-470.

Hill, R. W., Huelsman, T. J., Furr, R. M., Kibler, J., Vicente, B. B., \& Kennedy, C. (2004). A new measure of perfectionism: The Perfectionism Inventory. Journal of Personality Assessment, 82, 80-91.

Huelsman, T. J., Furr, R. M., \& Nemanick, R. C. (2003). Measurement of dispositional affect: Construct validity and convergence with a circumplex model of affect. Educational and Psychological Measurement, 63, 655-673.

Huelsman, T. J., Nemanick, R. C., \& Munz, D. C. (1998). Scales to measure four dimensions of dispositional mood: Positive energy, tiredness, negative activation, and relaxation. Educational and Psychological Measurement, 58, 804-819.

Molnar, D. S., Reker, D. L., Culp, N. A., Sadava, S. W., \& DeCourville, N. H. (2006). Journal of Research in Personality, 40, 482-500.

Pavot, W., \& Diener, E. (1993). Review of the satisfaction with life scale. Psychological Assessment, 5, 164-172.

Rice, K. G., Ashby, J. S., \& Slaney, R. B. (1998). Self-esteem as a mediator between perfectionism and depression: A structural equation analysis. Journal of Counseling Psychology, 45, 304-314.

Ryff, C. D. (1989). Happiness is everything, or is it? Explorations on the meaning of psychological well-being. Journal of Personality and Social Psychology, 57, 1069-1081.

Shafran, R., \& Mansell, W. (2001). Perfectionism and psychopathology: A review of research and treatment. Clinical Psychology Review, 21, 876-906.

Stoeber, J., \& Otto, K. (2006). Positive conceptions of perfectionism: Approaches, evidence, challenges. Personality and Social Psychology Review, 10, 295-319.

Stumpf, H., \& Parker, W. D. (2000). A hierarchical structural analysis of perfectionism and its relation to other personality characteristics. Personality and Individual Differences, 28, 837-852.

Tabachnick, B. G., \& Fidell, L. S. (2007). Using multivariate statistics (5th ed.). Boston, MA: Allyn and Bacon/Pearson Education.

Terry-Short, L. A., Owens, R. G., Slade, P. D., \& Dewy, M. E. (1995). Positive and negative perfectionism. Personality and Individual Differences, 18, 663-668. 